\title{
Effect of Barkhausen Detection Distance in Cold and Temper Rolled Low Carbon Steels: A Novel Approach
}

\author{
Mohamed M. Blaow ${ }^{1}$, Mohamed Ali Ballem² \\ ${ }^{1}$ Department of Industrial Engineering, Faculty of Engineering, Misurata University, Misurata, Libya \\ ${ }^{2}$ Department of Materials Science and Engineering, Faculty of Engineering, Misurata University, Misurata, Libya \\ Email: *mblaow@eng.misuratau.edu.ly, Mohamed.Ballem@eng.misuratau.edu.ly
}

How to cite this paper: Blaow, M.M. and Ballem, M.A. (2019) Effect of Barkhausen Detection Distance in Cold and Temper Rolled Low Carbon Steels: A Novel Approach. Journal of Materials Science and Chemical Engineering, 7, 1-11.

https://doi.org/10.4236/msce.2019.710001

Received: August 13, 2019

Accepted: October 9, 2019

Published: October 12, 2019

Copyright $\odot 2019$ by author(s) and Scientific Research Publishing Inc. This work is licensed under the Creative Commons Attribution International License (CC BY 4.0).

http://creativecommons.org/licenses/by/4.0/

\begin{abstract}
In this paper, a novel arrangement for magnetic Barkhausen noise detection is introduced. Measurements have been performed using two low carbon steel plates of $1 \mathrm{~mm}$ thickness. The measurements were conducted along the rolling and the transverse directions. The new arrangement includes a displacement of the detection coil in predetermined steps in while the magnetizing yoke is kept stationary introducing a cyclic magnetization in the rolling direction and transverse to it. In general, the intensity of the Barkhausen signals decreased as a function of coil displacement in both plates. In the temper rolled plate, Barkhausen noise profile shape changed from a single peak to a double peak one when coil has been displaced by $5 \mathrm{~mm}$ away in both magnetizing directions. Peaks are more apparent while magnetizing in the transverse direction. The appearance of two peaks profile in the temper rolled plate may be attributed to two stages of magnetization taking place at different times as a function of the applied field. Magnetization in the transverse direction results in a partition of the internal magnetizations into two main components perpendicular to each other. The internal components of magnetization involve the magnetic easy axes in the rolling direction and the forced magnetization in the transverse direction due to the applied field. Another assumption to interpret the findings may be due to the internal demagnetization field in the soft material below surface. The findings support this assumption in such a way that the demagnetizing field is strong enough in the transverse direction than in the rolling direction. This assumption is supported by the experiment on cold rolled plate. In the cold rolled plate, the resultant MBN profiles are composed of one peak throughout the test due to high dislocation density and hence a very weak demagnetizing field.
\end{abstract}




\section{Keywords}

Temper Rolled, MBN Profile, Internal Magnetization, Demagnetizing Field

\section{Introduction}

The discrete changes in local magnetization that results can be detected as voltage pulses in a search coil or magnetic read head. It is well known that, magnetic properties are affected by such factors as microstructure, heat treatment, chemical composition and mechanical condition [1]. Magnetic Barkhausen noise, $\mathrm{MBN}$ is produced by the irreversible movement of domain walls in a magnetization cycle. Domain walls are pinned temporarily by microstructural inhomogeneities and then released in the increasing magnetic field [2] [3]. This makes magnetic methods like Barkhausen noise technique ideal for the evaluation of condition of steel structures and components after various treatments.

Palit et al. [4] reported that the peak amplitude of Barkhausen emission is maximum along rolling direction (RD) and minimum along the transverse direction (TD) in AISI 304 stainless steel in both cold rolled and cold rolled and annealed condition. This indicates that magnetic easy axis predominantly lies along the RD. Kraus et al. [5] measured MBN signal as a function of the angle of the magnetic sweep field applied parallel to a sample's surface to investigate the relative magnitude and direction of the magnetic easy axis in pipeline steels [6] and in 3\% Si-Fe steel laminates [7]. They found that MBN energy is highest from the magnetic easy axis and decreases in the transverse direction.

To determine the effect of the mode of magnetization on the pattern of MBN signal, Bhattacharya and Vaidyanathan [8] performed some experiments on specimens of $2.25 \mathrm{Cr}-1 \mathrm{Mo}$ steel in an austenitized and air cooled condition magnetized by a yoke and a solenoid coil. They found that when the magnetization was performed by a yoke in closed circuit mode that the MBN signal composes of one single activity. However, when the magnetization was performed using a solenoid the MBN activity showed two peaks and a tilt in the B-H loop.

In this paper and based on the above mentioned findings, a novel way of MBN detection is introduced. The magnetization has been performed by a yoke but the detection coil was displaced in steps of $10 \mathrm{~mm}$ away up to $150 \mathrm{~mm}$ from the yoke each time in the rolling and the transverse directions.

\section{Materials and Method}

The experiment was carried out using $200 \times 200 \times 1 \mathrm{~mm}$ specimens of low carbon (AISI 1010) steel in the form $1 \mathrm{~mm}$ in annealed and stressed (temper rolled) condition and a cold rolled plate from the same steel grade. Apparatus used in experimental studies is described elsewhere [9]. The amplitude of the driving current to produce a maximum magnetic field strength of $4.5 \mathrm{kA} \cdot \mathrm{m}^{-1}$ at a frequency of $1 \mathrm{~Hz}$ is $1 \mathrm{~A}$. A relatively low excitation frequency was used to minim- 
ize eddy current opposition to the applied magnetic field, to ensure a relatively slow magnetization rate in the sample and high signals to noise ratio. Barkhausen noise emission was detected by an inductive search coil of 1000 turns of 0.1 $\mathrm{mm}$ insulated copper wire wounded around an empty cored $20 \mathrm{~mm}$ diameter cylinder. The signals were amplified to $40 \mathrm{~dB}$ and filtered with a $1-100 \mathrm{kHz}$ band pass filter in two stages. The magnetization was applied in the rolling direction and transverse to it. In each stage, some MBN measurements were conducted while the pick up coil is place at the yoke followed by a displacement of the pick up coil away in steps as shown in Figure 1.

MBN emission, to a first approximation, was correlated with the differential permeability of the material [3]. It follows that in a homogeneous material the emission is a maximum twice in each hysteresis loop. The intensity of the MBN emission is anticipated to peak at a positive field with increasing energizing current and peak again at a negative field as the state of magnetization of the sample moves around the $\mathrm{BH}$ loop [9].

In the first part of the experiment, directional MBN measurements were performed where the detection coil was placed between the legs of the magnetizing yoke in both specimens to investigate the magnetic anisotropy due to rolling. In the second part, the new approach of measuring indirect MBN by displacing the detection coil from the yoke. A measurement line in the middle of both specimens was identified and as a guide for the detection coil displacement during the experiment and the magnetizing yoke was placed at the plate end. The first point was identified where the detection coil was integrated with the magnetic yoke (point $0 \mathrm{~mm}$ ) then the detection coil was displaced in steps and the corresponding MBN signals were recorded. To increase the accuracy, $10 \mathrm{MBN}$ signals were recorded and averaged for every measurement point.

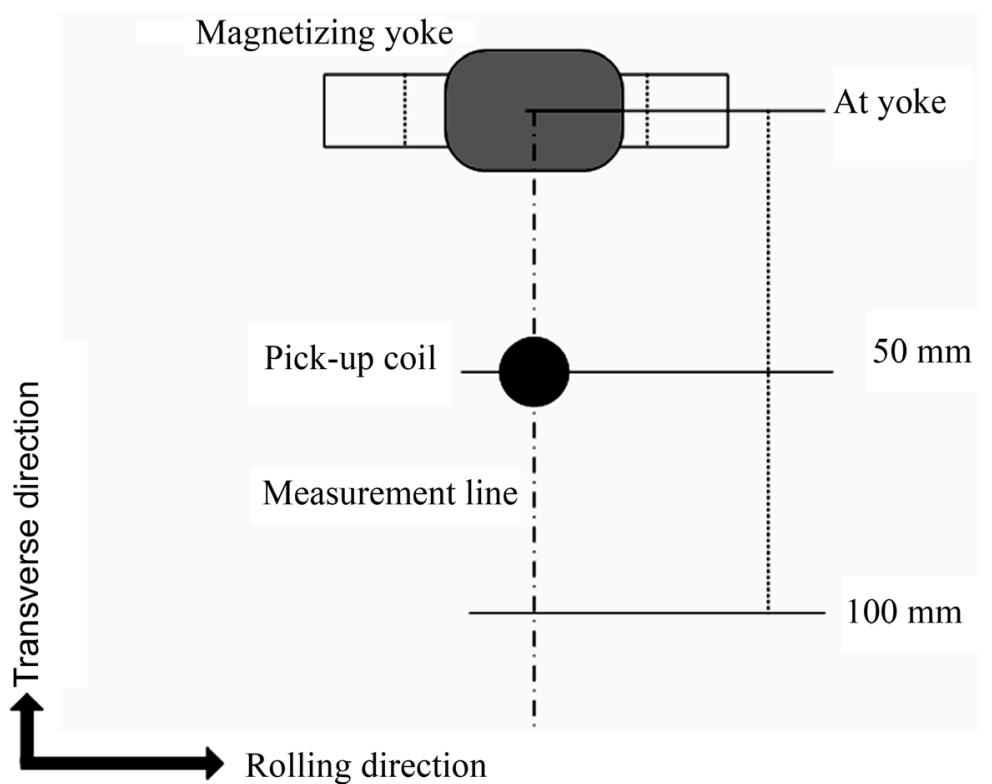

Figure 1. One-magnetization cycle MBN profiles where the detection coil is integrated with the yoke. 


\section{Results}

\subsection{Effect of Stress Status}

Example of the effect of residual stress condition in the temper rolled and cold rolled plates is shown by MBN profiles in Figure 2.

It can be seen that the MBN output from the temper rolled is higher than that of the cold rolled due to the difference in the dislocation densities in both plates.

\subsection{Effect of Detection Location}

In order to explore the effect of a gap between the magnetizing yoke and the MBN detection probe, the yoke was fixed at a point at the edge of the plate whereas the detection coil was displaced in steps. The induced MBN signal shape changes showing a double MBN activity as the gap was introduced in the detection arrangement as shown by time domain signal in Figure 3 in the temper rolled plate.

The result of indirect MBN measurements at the rolling direction is shown in Figure 4. The result shows that MBN profile changes in the first measurement at
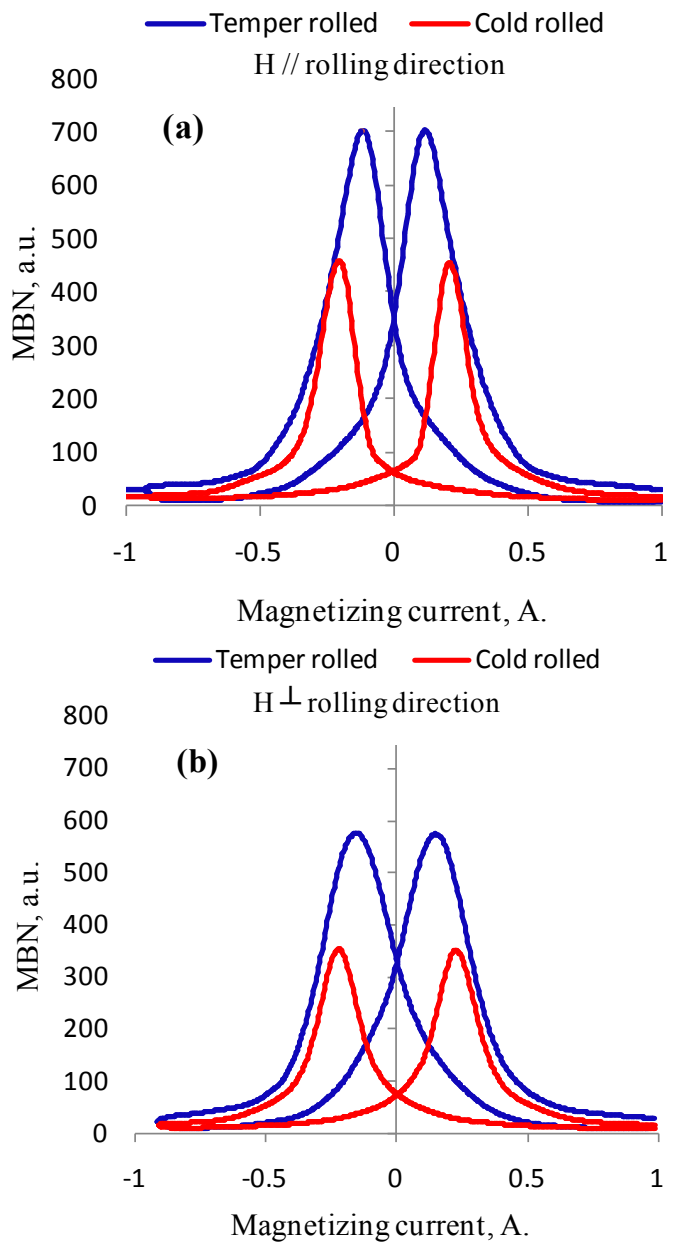

Figure 2. One-magnetization cycle MBN profiles where the detection coil is integrated with the yoke. 

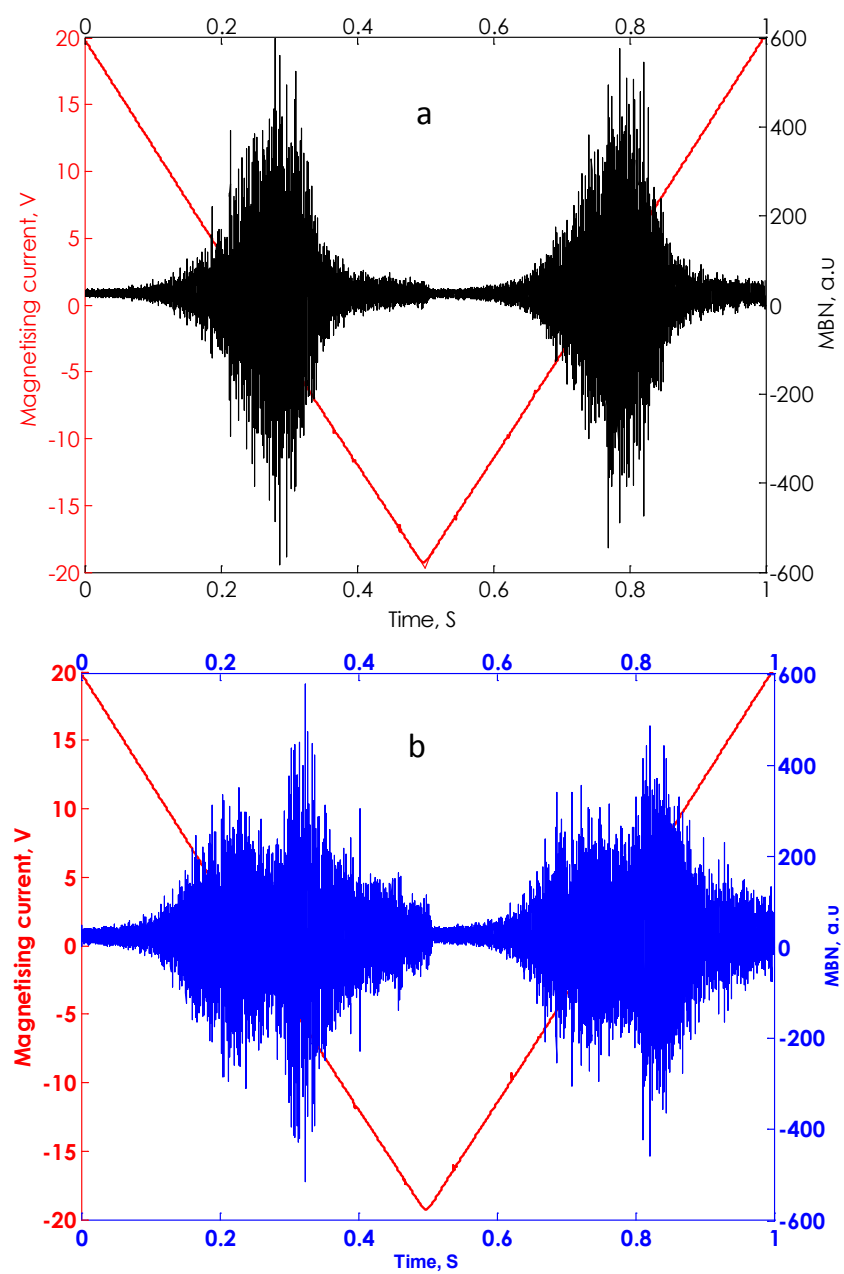

Figure 3. MBN time domain signals as a function of magnetizing direction and detection location from the temper rolled plate transverse direction: (a) Pick-up coil at yoke; (b) Pick-up at $50 \mathrm{~mm}$ to yoke.

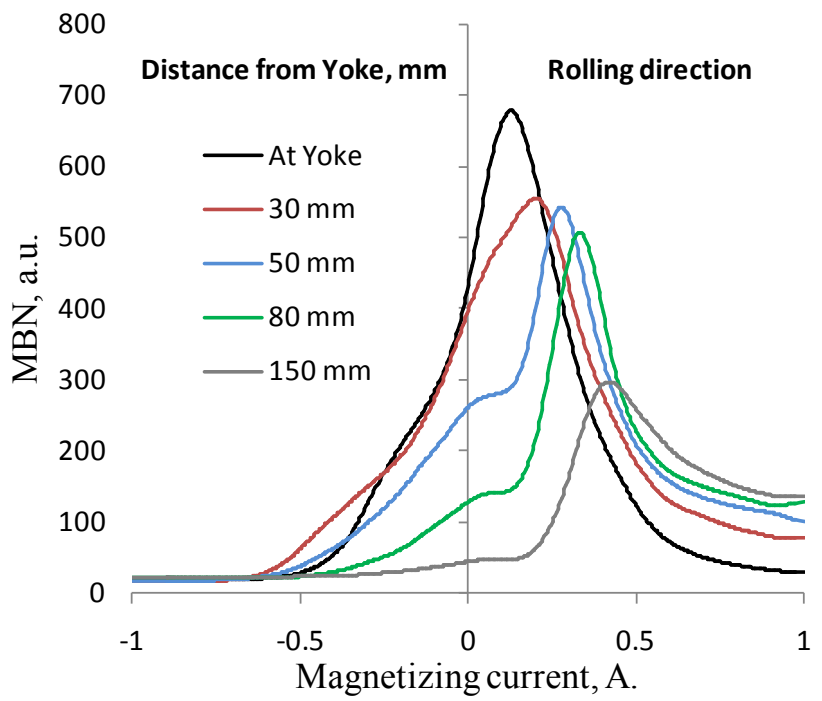

Figure 4. Half-magnetizing cycle profiles as a function of detection distance in the rolling direction $(\mathrm{RD})$. 
$5 \mathrm{~mm}$ from the yoke by showing broadening of the peak. This behavior develops by moving the detection probe further distance away from the yoke by a profile distortion. The complex profile indicates a minor peak at low field and a major second peak at higher field. This behavior continues for some distance in the magnetized landscape.

Barkhausen measurements were conducted in the transverse direction in similar manner. The result is shown in Figure 5. Figure 5 shows that the result is similar to that of the rolling direction except that the split of the MBN profile is sharper where a clear separation of two MBN activities is observed. This behavior continues until the first peak at low field diminishes at a gap of $150 \mathrm{~mm}$.

However, this is not the case in the cold rolled plate where a single peak profile was seen in each location of the detection coil as shown in Figure 6. The result from the cold rolled plate also show that the peak position was shifted to a higher field along with a decrease in the MBN intensity as a function detection displacement.

\section{Discussion}

Double peaks in MBN envelopes have been observed in a number of instances. In some cases, the double peaks can be ascribed to inhomogeneity in the material, as might arise in case carburized steel. In effect, the MBN measurement here can be seen as sampling a composite specimen. In other cases, double peaks have been observed only when stress is applied [10]. There is difficulty in understanding how the double peak arises in the latter case. If the rule that MBN output is proportional to $\mathrm{dB} / \mathrm{dH}$, the implication is that the $\mathrm{BH}$ curve is distorted by the application of stress as illustrated in the schematic presented in Figure 7,

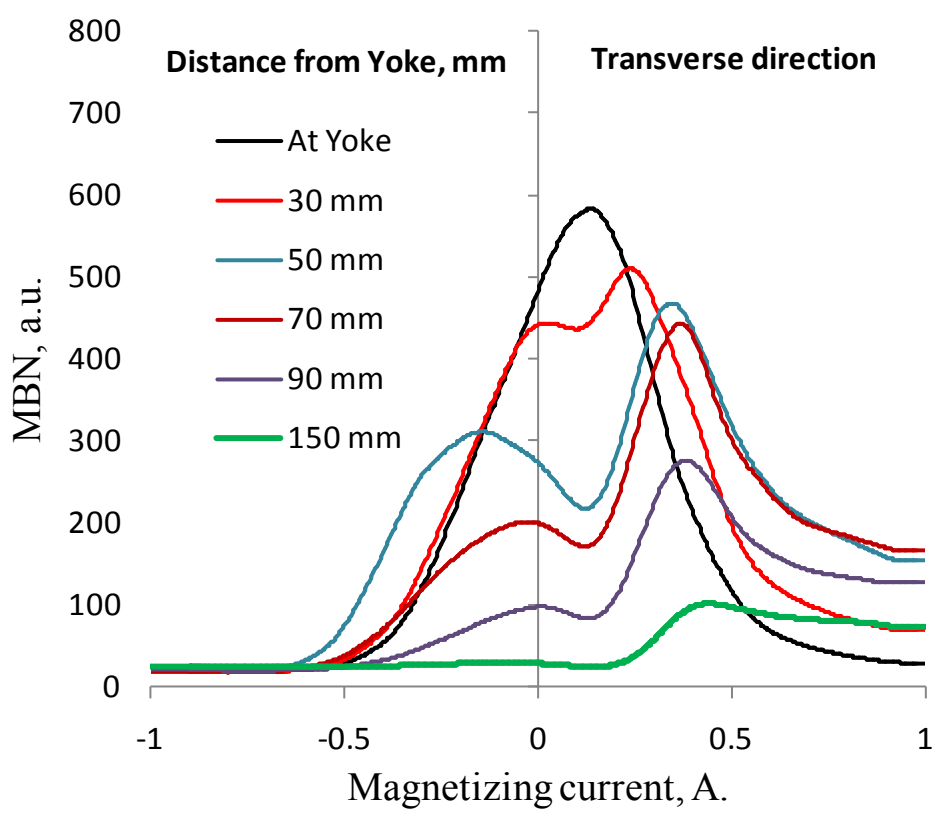

Figure 5. Half-magnetizing cycle profiles as a function of detection distance in the transverse direction (TD). 

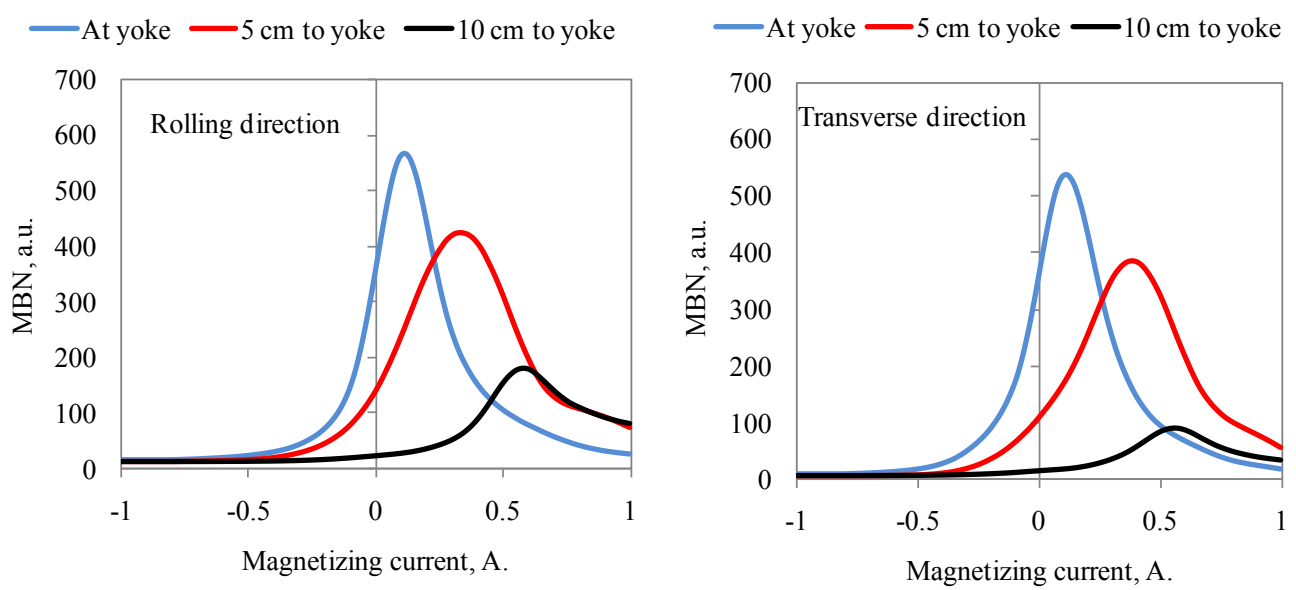

Figure 6. Half cycle MNB profiles as a function of pick-up location from the cold rolled plate.

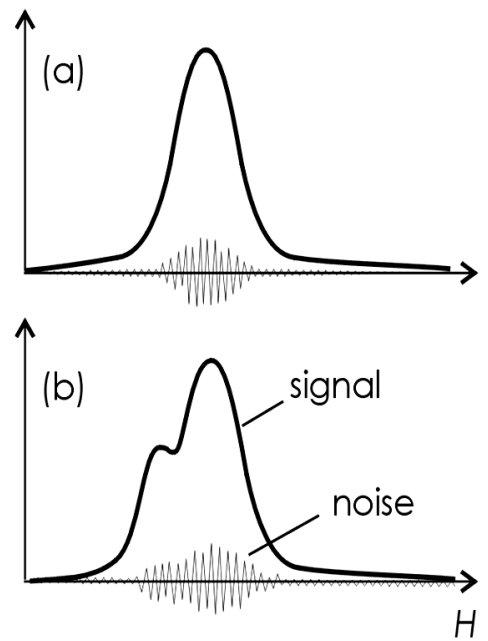

Figure 7. Schematic illustration of distortion in the BH curve inferred from the observation of double MBN peaks.

which shows that when a single peak is observed the BH curve is uniform (a), but in case of double MBN peaks the BH curve is distorted (b). The only other interpretation is that the double MBN peaks are, to some degree, independent of the $\mathrm{dB} / \mathrm{dH}$ versus $\mathrm{H}$ curve. There is some evidence that in some cases double peaks can occur at field strengths that do not coincide with the peak in $\mathrm{dB} / \mathrm{dH}$ versus $H$ curve [11] [12]. Evidence for this ought to be sought experimentally, but doing so might be difficult because of the fact that $\mathrm{dB} / \mathrm{dH}$ versus $\mathrm{H}$ behavior is representative of the bulk whereas MBN is associated with the surface layers.

Since, Hwang and Kim [13] have observed a three-peak MBN profile in unstrained mild steel. The two outer peaks were ascribed to nucleation and annihilation of reverse spike domains in the knee regions of the hysteresis loop. It was supposed that few spike domains were retained when the specimen was saturated, so that reversal of the field caused abrupt nucleation of new domains. It is not clear how these ideas can be reconciled with the present observations of the behavior of temper rolled plate. They produced evidence that the differential 
permeability is not coupled to MBN output in mild steel. They measured MBN output at the same time as measuring the B-H curve. A double peak MBN envelope was obtained in annealed samples, but the peaks did not coincide with the peak in the differential permeability.

In the literature there is an attempt of interpretation of the split of the MBN profile in the presence of a gap between the magnetizing unit and the detection coil was that presented by Bhattacharya and Vaidyanathan [8] who attributed it to the presence of a demagnetizing field. Based on that interpretation, the result here may suggest that the demagnetizing field is present in the plate and it is more effective in locations away from the magnetizing unit.

Yardly, V. [14] proposed a model (Equation (1)) to interpret BN data obtained experimentally from tempered steel [15]

$$
V\{H\}=C \cdot \sum_{i=1}^{m} \frac{A_{i} \sqrt{\pi}}{2(\Delta S)_{i}} \cdot \operatorname{erfc} \cdot\left\{\frac{H-\langle S\rangle_{i}}{(\Delta S)_{i}}\right\}
$$

where:

$V\{H\}$ : BN voltage at field $H$;

$C$ : Constant;

$S$ : Pinning site field strength;

$A_{i}$ : Total number pinning points of $i$ th type per unit volume;

$\langle S\rangle_{i}$ : Mean value of $S$ for $i$ th type of pinning site;

$(\Delta S)_{i}$ : Standard deviation of $S$ for $i$ th type of pinning site.

It was shown that plots of the function $V\{H\}$ for two pining strength values $(m=2)$ were able to fit with the experimental when appropriate values of the parameters $A_{i},\langle S\rangle_{i}$ and $\Delta S_{i}$ where selected. The result is shown in Figure 8.

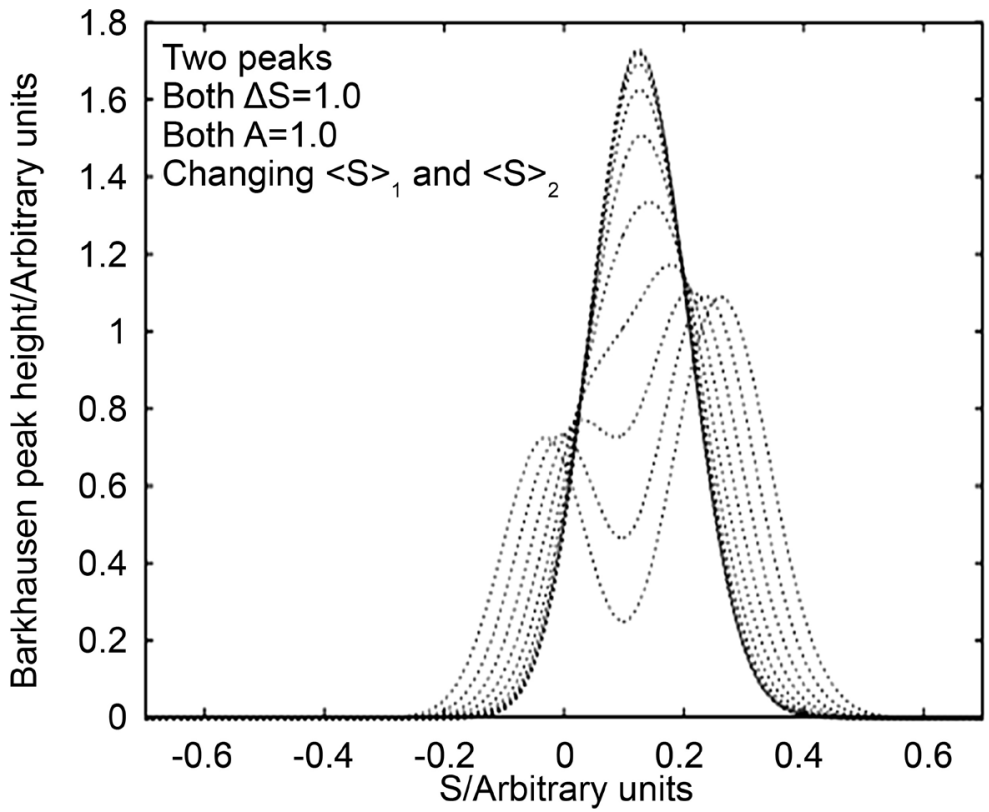

Figure 8. Transition between single and complex behavior [14]. 
According to [14], in order to test whether the model has any physical basis, the fitting parameters obtained were compared with grain and carbide size data from the work of Moorthy et al. [15]. In the Model, the parameters $\langle S\rangle_{1}$ and $\langle S\rangle_{2}$ represent the average pinning strengths of the two distributions. A model using a log-normal distribution of pinning site strengths for the lower-field peak, and a normal distribution for the higher-field peak, gave good agreement with real data. Clear relationships were found between the fitting parameters characterizing the centers of the distributions, and measured grain and carbide dimensions. It therefore appears that this model both supports the interpretation of Moorthy et al. [15] that two-peak BN signals are due to the separate effects of pinning by grain boundaries and carbides, and provides a basis for microstructural estimation from Barkhausen data.

In this paper, the material specifications were unaltered and that the only variable in the experiment was the detection distance on MBN signals. The transition of MBN behavior from a single peak profile to a double peak profile was caused by the displacement of the detection coil in the temper rolled steel. A proposed interpretation to the transition is such that when the detection coil is a part from the yoke, the effective internal field is at a threshold value that is needed to force domain walls to unpin from two different obstacle strengths in the temper rolled plate. Since the temper rolled plate is made a sandwich like bulk where the surfaces consist of deformed grains and the subsurface consists of fully annealed grains. It is expected that when the detection coil is located at the magnetizing yoke, the magnetization is guided by the applied field which is stronger than the threshold internal field and hence, a single MBN activity takes place. Away from the magnetizing yoke, the internal field experienced by the plate matrix is weakened by the demagnetizing field. A weak but enough to map the matrix, the internal field seems appropriate to map both types of grains especially when the magnetic field is applied in the transverse direction.

\section{Conclusions}

The magnetic Barkhausen noise method was used to qualitatively study the effect of detection spacing with angular excitation in textured steel. This work presents a novel approach in for MBN detection. The following points are addressed:

1) Direct $M B N$ measurements show that the highest $M B N$ peak is seen with the magnetization in the rolling direction and lowest is seen with the transverse direction due to the effect of anisotropy as a result of rolling in both plates.

2) In the temper rolled plate, the resultant $M B N$ is broadened as soon as the detection coil is displaced from the magnetizing yoke. On further displacement, the profile splits into two peaks. This behavior continues up unit the signal diminishes. This behavior is seen while magnetizing in the transverse direction whereas it is minor in the rolling direction. The cold rolled plate did not show this behavior because of the high dislocation density in the plate. 
3) Dislocations are effective barriers to domain walls movement and the magnetization process does not split. It seems that in high dislocation density the magnetization takes place randomly with no indication of favorable direction to follow. This implies that the resultant MBN is of a single activity.

4) The findings indicate that this novel way of MBN detection may map more details in the magnetized landscape in some materials. Also, MBN profile analysis is more efficient tool to indicate the material condition.

\section{Conflicts of Interest}

The authors declare no conflicts of interest regarding the publication of this paper.

\section{References}

[1] Jiles, D.C. (1988) The Influence of Size and Morphology of Eutectoid Carbides on the Magnetic Properties of Carbon Steels. Journal of Applied Physics, 63, 29802982. https://doi.org/10.1063/1.340921

[2] Jiles, D.C., Sipahi, L.B. and Williams, G. (1993) Modeling of Micromagnetic Barkhausen Activity Using a Stochastic Process Extension to the Theory of Hysteresis. Journal of Applied Physics, 73, 5830-5832. https://doi.org/10.1063/1.353541

[3] Jiles, D. (2000) Dynamics of Domain Magnetization and the Barkhausen Effect. Czechoslovak Journal of Physics, 50, 893-924.

https://doi.org/10.1023/A:1022846128461

[4] Palit Sagar, S., Ravi Kumar, B., Dobmann, G. and Bhattacharya, D.K. (2005) Magnetic Characterization of Cold Rolled and Aged AISI 304 Stainless Steel. NDT \& E International, 38, 674-681. https://doi.org/10.1016/j.ndteint.2005.04.004

[5] Krause, T.W., Mandal, K., Hauge, C., Weyman, P., Sijgers, B. and Atherton, D.L. (1997) Correlation between Magnetic Flux Leakage and Magnetic Barkhausen Noise: Stress Dependence in Pipeline Steel. Journal of Magnetism and Magnetic Materials, 169, 207-219. https://doi.org/10.1016/S0304-8853(96)00714-7

[6] Krause, T.W., Clapham, L. and Atherton, D.L. (1994) Characterization of the Magnetic Easy Axis in Pipeline Steel Using Magnetic Barkhausen Noise. Journal of Applied Physics, 75, 7983-7988. https://doi.org/10.1063/1.356561

[7] Krause, T.W., Szpunar, J.A., Birsan, M. and Atherton, D.L. (1996) Correlation of Magnetic Barkhausen Noise with Core Loss in Oriented 3\% Si-Fe Steel Laminates. Journal of Applied Physics, 79, 3156-3167. https://doi.org/10.1063/1.361258

[8] Bhattacharya, D.K. and Vaidyanathan, S. (1997) Effect of the Demagnetisation Factor on the Barkhausen Noise Signal. Journal of Magnetism and Magnetic Materials, 166, 111-116. https://doi.org/10.1016/S0304-8853(96)00444-1

[9] Blaow, M., Evans, J. and Shaw, B. (2005) Surface Decarburisation of Steel Detected by Magnetic Barkhausen Emission. Journal of Materials Science, 40, 5517-5520. https://doi.org/10.1007/s10853-005-4240-5

[10] Kleber, X. and Vincent, A. (2004) On the Role of Residual Internal Stresses and Dislocations on Barkhausen Noise in Plastically Deformed Steel. NDT \& E International, 37, 439-445. https://doi.org/10.1016/j.ndteint.2003.11.008

[11] Sablik, M.J. (1993) A Model for the Barkhausen Noise Power as a Function of Applied Magnetic Field and Stress. Journal of Applied Physics, 74, 5898-5900. https://doi.org/10.1063/1.354167 
[12] Sablik, M.J. and Augustyniak, B. (1996) The Effect of Mechanical Stress on a Barkhausen Noise Signal Integrated across a Cycle of Ramped Magnetic Field. Journal of Applied Physics, 79, 963-972. https://doi.org/10.1063/1.360880

[13] Hwang, D.G. and Kim, H.C. (1988) The Influence of Plastic Deformation on Barkhausen Effects and Magnetic Properties in Mild steel. Journal of Physics D: Applied Physics, 21, 1807-1813. https://doi.org/10.1088/0022-3727/21/12/024

[14] Yardley, V.A. (2003) Magnetic Detection of Microstructural Change in Power Plant Steels. Ph.D. Thesis. University of Cambridge, Cambridge.

[15] Moorthy, V., Vaidyanathan, S., Jayakumar, T. and Raj, B. (1998) On the Influence of Tempered Microstructures on Magnetic Barkhausen Emission in Ferritic Steels. Philosophical Magazine A, 77, 1499-1514.

https://doi.org/10.1080/01418619808214266 